\title{
TDP-43 pathology in the basal forebrain and hypothalamus of patients with amyotrophic lateral sclerosis
}

Matthew D Cykowski ${ }^{1}$, Hidehiro Takei ${ }^{1,2}$, Paul E Schulz ${ }^{3,4}$, Stanley H Appel ${ }^{2,5}$ and Suzanne Z Powell ${ }^{1,2^{*}}$

\begin{abstract}
Introduction: Amyotrophic lateral sclerosis is a neurodegenerative disease characterized clinically by motor symptoms including limb weakness, dysarthria, dysphagia, and respiratory compromise, and pathologically by inclusions of transactive response DNA-binding protein $43 \mathrm{kDa}$ (TDP-43). Patients with amyotrophic lateral sclerosis also may demonstrate non-motor symptoms and signs of autonomic and energy dysfunction as hypermetabolism and weight loss that suggest the possibility of pathology in the forebrain, including hypothalamus. However, this region has received little investigation in amyotrophic lateral sclerosis. In this study, the frequency, topography, and clinical associations of TDP-43 inclusion pathology in the basal forebrain and hypothalamus were examined in 33 patients with amyotrophic lateral sclerosis: 25 men and 8 women; mean age at death of 62.7 years, median disease duration of 3.1 years (range of 1.3 to 9.8 years).

Results: TDP-43 pathology was present in 11 patients (33.3\%), including components in both basal forebrain $(n=10)$ and hypothalamus $(n=7)$. This pathology was associated with non-motor system TDP-43 pathology $\left(X^{2}=17.5\right.$, $p=0.00003)$ and bulbar symptoms at onset $\left(X^{2}=4.04, p=0.044\right)$, but not age or disease duration. Furthermore, TDP-43 pathology in the lateral hypothalamic area was associated with reduced body mass index $(W=11, p=0.023)$.

Conclusions: This is the first systematic demonstration of pathologic involvement of the basal forebrain and hypothalamus in amyotrophic lateral sclerosis. Furthermore, the findings suggest that involvement of the basal forebrain and hypothalamus has significant phenotypic associations in amyotrophic lateral sclerosis, including site of symptom onset, as well as deficits in energy metabolism with loss of body mass index.
\end{abstract}

Keywords: Amyotrophic lateral sclerosis, TDP-43, Basal forebrain, Hypothalamus

\section{Introduction}

Amyotrophic lateral sclerosis (ALS) is a progressive neurodegenerative disease characterized by loss of upper and/or lower motor neurons, muscle wasting, and bulbar symptoms (e.g., dysarthria, dysphagia) [1]. The pathologic underpinnings of upper and lower motor neuron deficits in ALS include neuronal loss and gliosis in large neurons of "canonical" regions, including motor cortex, lamina IX of the ventral horn of the spinal cord, and somatic motor cranial nerve nuclei of the brainstem (XII, IX, and V) [2].

\footnotetext{
* Correspondence: spowell@houstonmethodist.org

'Department of Pathology and Genomic Medicine, Houston Methodist

Hospital, 6565 Fannin Street, Suite M227, Houston, TX 77030, USA

${ }^{2}$ Houston Methodist Neurological Institute, Houston Methodist Hospital,

Houston, TX, USA

Full list of author information is available at the end of the article
}

Detailed pathologic studies have also demonstrated surprisingly widespread, non-motor pathology in some ALS patients, typically in the form of ubiquinated cytoplasmic inclusions that are immunoreactive for transactivating responsive sequence (TAR) DNA-binding protein $43 \mathrm{kDa}$, or TDP-43 [3]. Pathologic TDP-43 deposition is presumed to induce neuronal dysfunction through the cytoplasmic accumulation of toxic C-terminal TDP-43 fragments, or alternately, via the loss of constitutively expressed nuclear TDP-43 that is critical in transcriptional regulation and RNA processing [4]. As such, widespread TDP-43 proteinopathy in "non-canonical" brain regions (striatum, amygdala, non-frontal cortex, hippocampus) may underlie multifocal neuronal dysfunction that contributes 
to complex non-motor phenotypes in ALS, including cognitive impairment with prominent frontal executive dysfunction [5] and extrapyramidal signs [1]. Clinicopathologic studies confirming this presumption are rare, but those that have been performed do support an association between extra-motor TDP-43 pathology and extra-motor clinical findings [6].

Somewhat unique among extra-motor features in ALS are alterations in energy metabolism [7], autonomic function [8], and in some patients $[9,10]$ and animal models [11], involvement of the hypothalamic-pituitary axis. Deficits in energy metabolism are well recognized in ALS, with a paradoxical hypermetabolism occurring in many patients that combines with dysphagia to result in malnourishment and loss of body mass index (BMI) [12]. Along with advanced age and bulbar onset at diagnosis [13], loss of more than one BMI unit over two years is associated with a significantly shorter survival and more rapid disease progression [7]. Deficits in energy metabolism and autonomic function in ALS suggest the possibility of hypothalamic dysfunction, which may be indicated by pathologic TDP-43 deposition. This is further supported by evidence from Alzheimer disease [14], argyrophilic grain disease [15], Lewy body spectrum diseases [16], and multiple system atrophy [17], in which neurons of the hypothalamic region and adjacent basal forebrain are susceptible to neurodegenerative diseaseassociated cellular injury [18]. Although similar forebrain/hypothalamic pathology may be present in ALS and contribute to the extra-motor features of the disease $[1,8]$, this has yet to be systematically investigated.

This study was carried out to determine whether the basal forebrain and hypothalamus are pathologically involved in ALS. We hypothesized that pathologic TDP43 inclusions would be identified in a subset of ALS patients given the deficits in energy metabolism and autonomic function in ALS and frequent involvement of these regions in other neurodegenerative diseases. The frequency and topography of TDP-43 inclusions were examined in 33 ALS patients within these structures. Pathology in this region also was examined with respect to disease duration, the extent of TDP-43 proteinopathy, last available BMI, which is an indirect measure of energy metabolism, and patient age, since TDP-43 pathology may be seen in older patients without ALS [19].

\section{Materials and methods}

Pathologic materials were reviewed for 56 consecutive patients with clinically diagnosed and pathologically confirmed ALS. Archival materials were screened for representative portions of basal forebrain and hypothalamus (defined further below). Thirty-three patients were selected for study based on the presence of appropriate anatomic regions.

\section{Autopsy and clinical data}

Patient autopsies performed at the Houston Methodist Hospital included sections from all cerebral lobes, basal ganglia, thalamus, amygdala, hippocampus, midbrain, pons, medulla, cerebellum, spinal cord and pituitary. Immunostains performed on the majority of cases included tau (Dako, polyclonal, 1:40,000 dilution), beta-amyloid (Dako, clone $6 \mathrm{~F} / 3 \mathrm{D}, 1: 20$ dilution), alpha-synuclein (EMD Millipore, clone AB5038, rabbit polyclonal, 1:4000), and TDP-43 (ProteinTech, 10782-2-AP, polyclonal antibody, 1:200). An immunostain for ubiquitin (Dako, polyclonal, 1:500 dilution) also was performed on several archived cases. Immunostaining was performed on brain tissue that was fixed in $20 \%$ formalin for a minimum of seven days following brain removal. Sections were further fixed in $10 \%$ formalin until processing and embedding. Formalin fixed paraffin embedded tissue was sectioned at 4 microns, mounted on plus coated slides and dried at $60^{\circ} \mathrm{C}$. Immunostaining was performed on the BenchMark ULTRA $^{\odot}$ platform (Ventana Medical Systems, Inc., Tucson, AZ) with appropriate positive and negative controls.

Neuropathologists at Houston Methodist Hospital (H.T., S.Z.P.) utilized established criteria to assess Alzheimer disease (AD) neuropathologic changes, including neurofibrillary changes [20,21] and parenchymal amyloid [22,23], Lewy body pathology [16], ALS pathology [24], cerebral amyloid angiopathy (CAA), and vascular brain injury (VBI). Clinical variables recorded included date of symptom onset and date of death (disease duration calculated as the difference between these), presenting symptoms, clinical dementia, and family history of ALS. Body mass index (BMI) was also recorded for patients where this data was available within $\leq 24$ months of death (29 of 33 patients, median interval of 3.6 months between measurement and death, interquartile range 0.8-8.6 months). These studies were carried out with the approval of the Institutional Review Board at Houston Methodist Hospital (IRB-2-0114-0013).

\section{Identification of basal forebrain and hypothalamic components}

Basal forebrain components were defined on $\mathrm{H} \& \mathrm{E}$ and TDP-43 stains and included ventral pallidum (VP), ventral striatum and small granule cell islands (VS/Is), the bed nucleus of the stria terminalis (BNST), lateral septal area (LSA), and other neurons of the substantia innominata (SI). VP included large pallidal neurons ventral to fibers of the anterior commissure [25]. SI included medium-sized neurons, which did not always respect anatomic borders, as well as magnocellular neurons (e.g., nucleus of the diagonal band, nucleus basalis) [26]. VS/Is included medium-sized striatal neurons and islands of small granule cells, located within or ventromedial to VS, as well as within SI and VP in the region of the 
olfactory tubercle [27]. Since the distinction between small granule cells comprising "Islands of Calleja" versus "interface" islands [28] is not clear, we adopted the descriptive term used here. BNST was defined as the wedge-shaped group of medium-sized neurons dorsal to anterior commissure, medial to the internal capsule, ventral to caudate nucleus, and lateral to the LSA [26,29].

Hypothalamic nuclear groups were defined on H\&E and TDP- 43 stains by cytologic characteristics and relative position using standard anatomic references [18,29-32]. Hypothalamic nuclei often merge with surrounding nuclear groups without distinct borders [29,30], due in part to inherent anatomic variability in the region [31]. Therefore, neuronal groups with similar positional and cytologic characteristics were grouped for recording of TDP-43 pathology. Nuclei examined at the chiasmatic level included discrete magnocellular neurosecretory neurons of the supraoptic $(\mathrm{SON})$ and paraventricular $(\mathrm{PaV})$ nuclei [30]. At the chiasmatic level, TDP-43 pathology in small- to medium-sized neurons of the preoptic area (POA) [31] (including "hypothalamic" or "chiasmatic gray" in some sources $[18,30]$ ) was recorded. At the tuberal and mammillary levels, neurons assessed included ventrally located, medium- to large-sized neurons of the medial mammillary nucleus, lateral tuberal nucleus (in some sections), and ventral portion of the tuberomammillary nucleus (MN/LTN/TMN). Likewise, large neurons lateral to the fornix and mammillothalamic tract were recorded as lateral hypothalamic area, which merges with the dorsal portion of the tuberomammillary nucleus (LHA/TMN) $[29,32]$. At the tuberal level, inclusions were also recorded in diffuse, small- to medium-sized neurons adjacent to the third ventricle, including those of the ventromedial hypothalamus (PVL/TBL/VMH). At the mammillary level, medial to fiber bundles of the mammillothalamic tract, medium- to large-sized periventricular neurons were recorded as posterior hypothalamic area (PHA) [31,32].

These anatomic regions are shown in schematic form for basal forebrain (Figure 1A), including LSA and BNST, and hypothalamus at the caudal chiasmatic/ tuberal level (Figure 1B), and level of the mammillary bodies (Figure 1C).

\section{Assessment of TDP-43 pathology}

Two authors (M.D.C., H.T.) independently rated TDP43 pathology in the aforementioned areas. The location of pathologic TDP-43 inclusions was recorded and classified by morphologic sub-type as follows: neuronal cytoplasmic (NCI), neuronal intranuclear (NNI), glial cytoplasmic (GCI), and dystrophic neurites. For cases with TDP-43 inclusion pathology, quantitative measures were performed in the area of greatest hypothalamic pathology (or basal forebrain in cases without hypothalamic pathology). For these cases, any cellular TDP-43
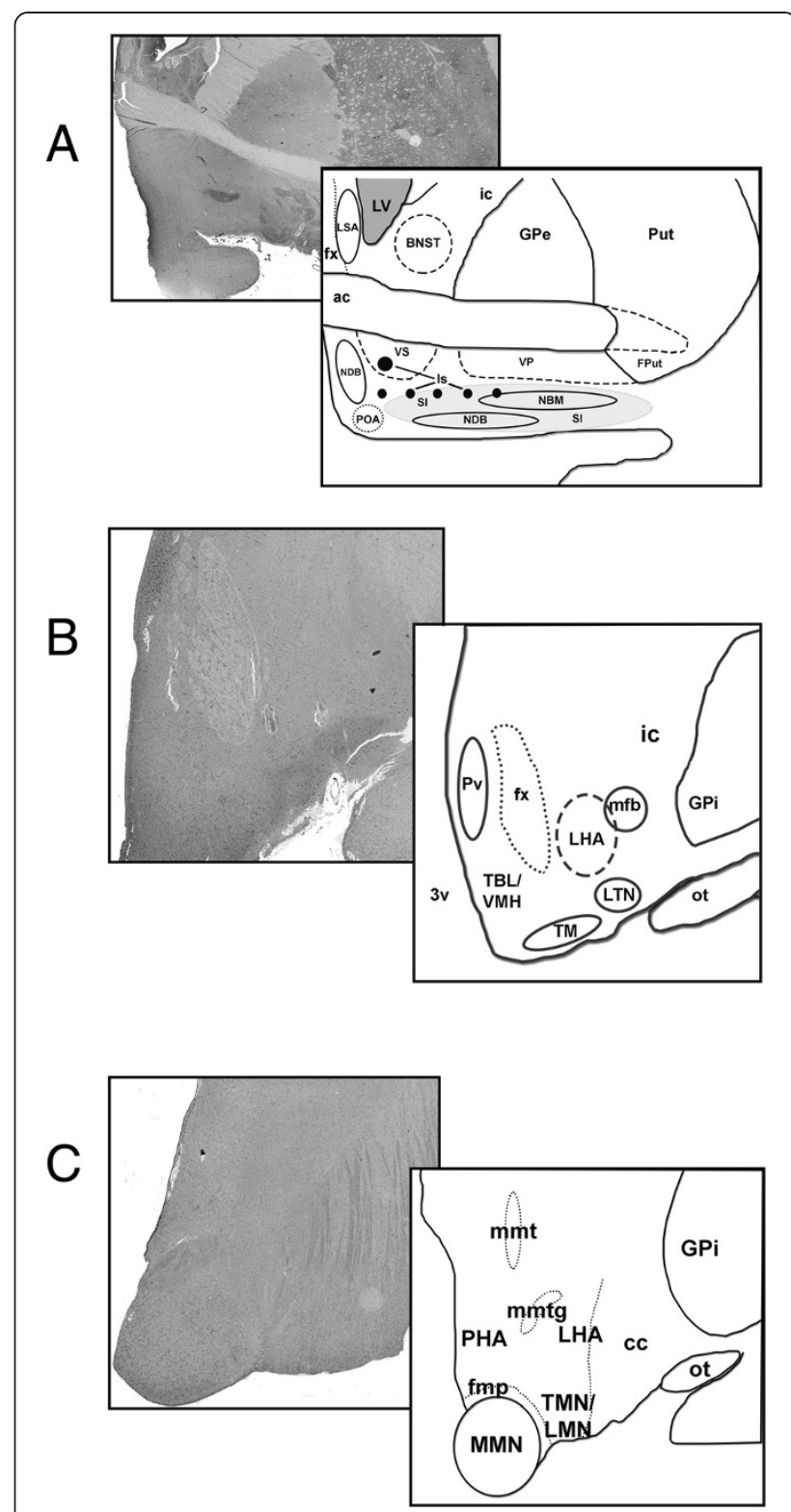

Figure 1 Schematic demonstrating anatomic structures investigated in the basal forebrain (A), mid-hypothalamus (tuberal level) (B), and caudal hypothalamus (mammillary level) (C) (see Materials and methods for detail). To the left of each schematic, grayscale renderings of TDP-43-stained sections of study patients are shown for comparison. In A, the continuity of rostral/chiasmatic hypothalamus and basal forebrain, as discussed in the text, is shown on both the histologic image (which also includes the optic chiasm) and on the schematic (POA). Abbreviations not defined in text: $a c$ - anterior commissure, $c c$ - crus cerebri, fmp - fasciculus mammillaris princeps (mammillary efferents), fx - fornix, GPe/i - external/ internal segments of the globus pallidus, FPut - fundus of the putamen, ic - internal capsule, $L V$ - lateral ventricle, $m f b$ - medial forebrain bundle, $m m t$ - mammillothalamic tract, $m m t g$ - mammillotegmental tract, ot - optic tract, $3 v$ - third ventricle. 
inclusions (NCI, GCI) were recorded within three consecutive high-power microscopic fields (HPF) at $400 \times$ magnification $\left(0.19625 \mathrm{~mm}^{2}\right.$ per HPF). Global TDP-43 pathologic burden was classified as canonical (brainstem, spinal cord, motor cortex) or non-canonical (canonical regions plus dorsal striatum, thalamus, non-frontal isocortex, and mesial temporal lobe).

\section{Statistical analysis}

Two-sided Mann-Whitney (Wilcoxon rank sum) testing was used to determine whether: (a) disease duration significantly differed between patients with and without basal forebrain/hypothalamic pathology, (b) disease duration significantly differed between patients with canonical and non-canonical TDP-43 pathology, (c) BMI significantly differed in patients with and without basal forebrain/hypothalamic pathology, (d) BMI significantly differed in patients with and without LHA pathology, and (e) age at death significantly differed between those with and without basal forebrain/hypothalamic TDP-43 pathology. Chi-squared $\left(\mathrm{X}^{2}\right)$ testing was used to determine whether basal forebrain/hypothalamic pathology was associated with (a) non-canonical TDP-43 pathology and (b) bulbar/respiratory onset.

\section{Results}

\section{Clinical characteristics and autopsy findings}

Table 1 lists demographic information for 33 ALS patients. Presenting symptoms included unilateral extremity weakness with or without muscle fasciculations (46.4\%), bulbar symptoms such as slurred speech, shortness of breath, dysarthria, and difficulty swallowing (25.0\%), muscle cramps $(17.8 \%)$, bilateral extremity or generalized weakness with/without fasciculations (17.8\%), falls $(10.7 \%)$, and foot drop (3.5\%). One patient showed clinically evident cognitive impairment with episodes of disorientation and word-finding difficulties. In two patients with a family history of ALS the genes implicated were not known (one of the two had SOD1, TARDBP, ANG, FUS, FIG4 studied) though both patients had ALS TDP-43 pathology outside of the hypothalamic region.

At autopsy, all patients had pathologically confirmed ALS. Table 1 lists non-ALS brain pathologies identified, including AD neuropathologic changes, CAA and VBI.

\section{TDP-43 inclusion pathology in basal forebrain and hypothalamus}

Pathology in basal forebrain and hypothalamus was present in 11 of 33 cases (33.3\%). This included components of basal forebrain in 10 patients: VS/Is, $n=9$ of 26 patients with the structure present; BNSTL, $\mathrm{n}=8$ of 21 ; SI neurons, $\mathrm{n}=6$ of 28 ; typically in medium-sized neurons with magnocellular neurons having filamentous/skein-like inclusions in only two cases; LSA, $\mathrm{n}=2$ of 15 ; and, VP,
Table 1 Demographics and non-ALS pathology in 33 patients

\begin{tabular}{ll}
\hline Average age at death (years) & $62.7(\mathrm{~s}=9.09)$ \\
Male/Female & $\mathrm{n}=25 / \mathrm{n}=8$ \\
Median duration (years) & 3.1 (interquartile range, 2.2-4.8) \\
Brain weight (grams) & 1348.6 ( $\mathrm{s}=156.97)$ \\
Any neurofibrillary pathology & $\mathrm{n}=24$ \\
Any parenchymal amyloid & $\mathrm{n}=9$ \\
Thal stage $\geq \|$ & $\mathrm{n}=7$ \\
Braak stage $\geq$ III $^{1}$ & $\mathrm{n}=4$ \\
CERAD $\geq$ "moderate" & $\mathrm{n}=2$ \\
CAA present & \\
VBI present & \\
LB pathology $^{3}$ & $\mathrm{n}=6$ \\
\hline Ab & $\mathrm{n}=3$
\end{tabular}

Abbreviations: CAA cerebral amyloid angiopathy, CERAD Consortium to Establish a Registry for Alzheimer Disease (see text), $L B$ Lewy body, VBI vascular brain injury.

Notes: 'One patient with Braak stage III had co-existing argyrophilic grain disease (AGD). ${ }^{2} \mathrm{CAA}$ was diffuse in only 1 of these 6 patients. ${ }^{3} \mathrm{VBI}$ was present in one patient each as remote infarct (left temporal lobe), hippocampal microinfarct, and acute/subacute hemispheric infarct.

$\mathrm{n}=1$ of 21 . This also included components of the hypothalamus in 7 patients, including LHA/TMH, $\mathrm{n}=5$ of 28; PHA, $\mathrm{n}=3$ of $24 ; \mathrm{MB} / \mathrm{LTN} / \mathrm{TMN}, \mathrm{n}=6$ of $19 ; \mathrm{PVN} / \mathrm{POA}$, $\mathrm{n}=2$ of 16 ; and PVL/TBL/VMH, $\mathrm{n}=4$ of 10 . The SON was not involved $(\mathrm{n}=13)$. Representative findings are demonstrated for basal forebrain and hypothalamus in Figures 2 and 3, respectively.

Neuronal inclusions were uniformly cytoplasmic (NCIs), while intranuclear inclusions were not identified. The density of TDP-43 cellular inclusions varied between 1/three HPFs to 72/three HPFs: the average number of inclusions was $8.42(\mathrm{sd}=18.75)$, with a per field average of 2.8 inclusions. NCI morphology consisted of granular preinclusions (e.g., Figure 3F), dense perinuclear and cytoplasmic inclusions that were crescent-shaped or globoid (e.g., Figure 2D and F, Figure 3B and E), and rarely, densely staining filamentous and skein-like inclusions (Figure 2B and inset of Figure 3B). GCIs were frequently identified in patients with NCIs in basal forebrain and hypothalamus. GCIs could be distinguished from small neurons with NCIs in the forebrain on the basis of cell size (see inset of Figure $2 \mathrm{H}$ for comparison). Thick, tortuous TDP-43 positive neurites were occasionally seen (see bottom left of Figure $2 \mathrm{H}$ ) but were uncommon.

For all cases positive for TDP-43 pathology in the regions under investigation $(\mathrm{n}=11)$, two authors (MDC, SZP) performed a post-hoc rating of gliosis (on H\&E stain) as absent, mild, moderate, or severe. One patient had mild gliosis in the preoptic area of the hypothalamus and this same patient had mild to focally moderate gliosis in basal forebrain (most apparent in the BNST). Three 


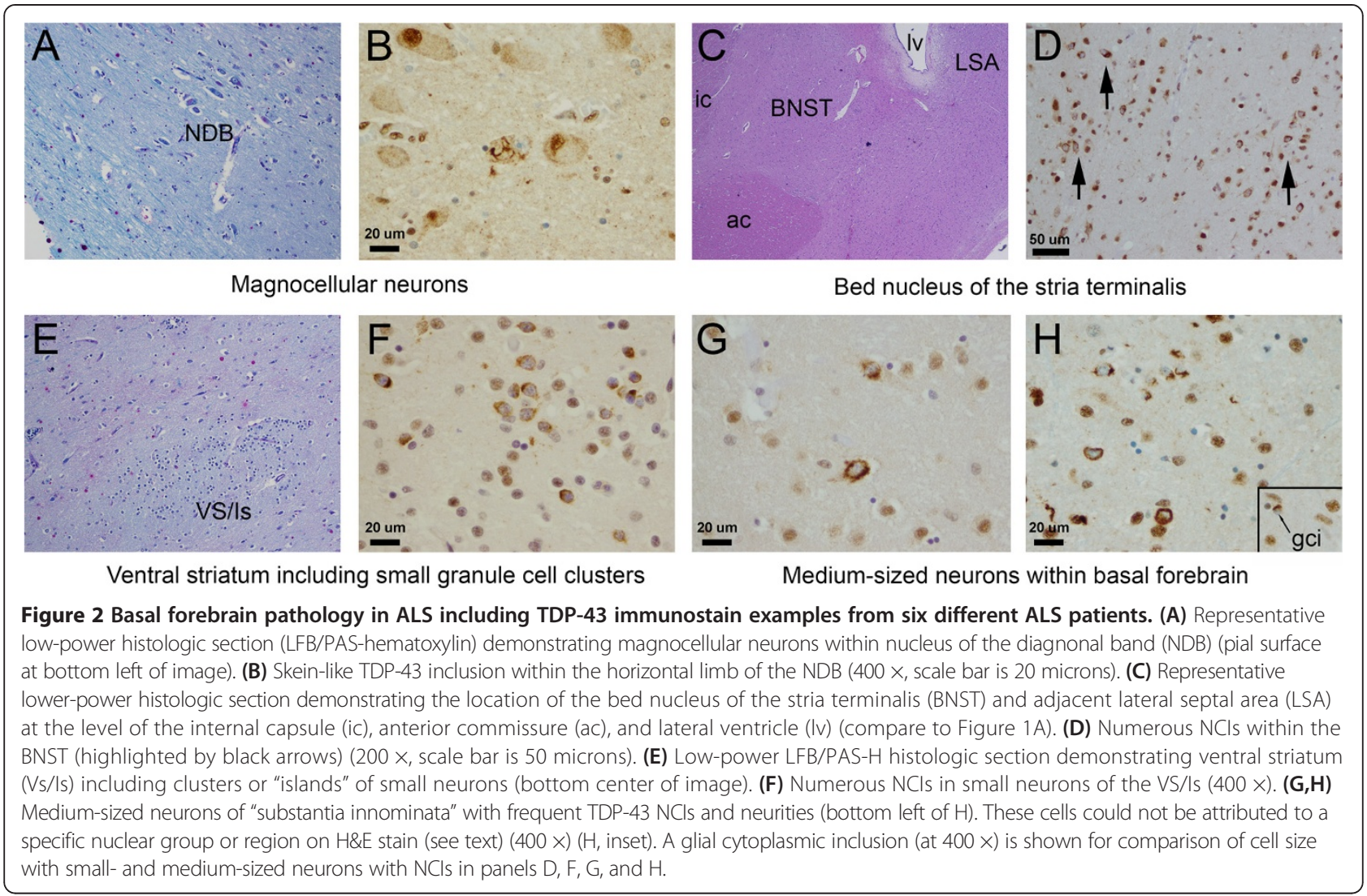

additional patients had mild gliosis in basal forebrain only, typically most apparent medially including within BNST. A fifth patient had moderate gliosis more diffusely throughout basal forebrain ventral to the anterior commissure (this patient had 63 TDP-43 cellular inclusions/three HPFs). Neuronal loss was not evident on H\&E stain in these patients.

\section{Extent of TDP-43 pathology}

ALS pathology in typical (canonical) regions was characterized by neuronal loss and gliosis with ubiquitinated or TDP-43 immunoreactive inclusions. Brain regions implicated included spinal cord $(\mathrm{n}=33)$, brainstem motor nuclei $(n=27)$, other brainstem nuclei $(n=17)$, and agranular frontal cortex $(n=22)$. Seventeen of 33 patients had pathologic involvement limited to these canonical regions. The remaining 16 patients had pathology in these regions plus non-canonical pathology, i.e. additional involvement of hippocampus $(\mathrm{n}=11)$, amygdala $(\mathrm{n}=11)$, non-frontal isocortex $(\mathrm{n}=9)$, dorsal striatum $(\mathrm{n}=10)$, and thalamus $(\mathrm{n}=8)$.

\section{Association of pathology in basal forebrain/hypothalamus with non-canonical pathology}

TDP-43 pathology in basal forebrain/hypothalamus was seen only with non-canonical pathology $\left(\mathrm{X}^{2}=17.5, \mathrm{p}=\right.$ 0.00003). Moreover, all but one case with hypothalamic
TDP-43 pathology occurred with concurrent basal forebrain pathology. In contrast, sparse basal forebrain pathology (range of 2-3 NCIs in three HPFs) occurred in three cases without hypothalamic pathology. An additional case with dense basal forebrain pathology (63 NCIs in three HPFs) did not have hypothalamic tissue available for study.

\section{Association of pathology with clinical presentation}

There was a significant association between bulbar onset symptoms and basal forebrain/hypothalamic pathology $\left(\mathrm{X}^{2}=4.04, \mathrm{p}=0.044\right)$ with $71.4 \%$ of all patients having bulbar symptoms at disease onset demonstrating this pathology (compared to $28.6 \%$ of patients without bulbar symptoms at onset).

\section{Association of pathology with disease duration and patient age}

For patients with complete data on disease duration $(\mathrm{n}=30)$, this parameter did not significantly differ between patients with basal forebrain/hypothalamic TDP-43 pathology (median of 3.26 years, interquartile range, 2.5-5.3) and without (median of 3.1 years, interquartile range, 2.1-3.5) ( $\mathrm{W}=90, \mathrm{p}=0.55)$. Likewise, the density of TDP-43 inclusions was not correlated to disease duration (Spearman rho $=0.18, \mathrm{p}=0.33$ ). Disease duration 


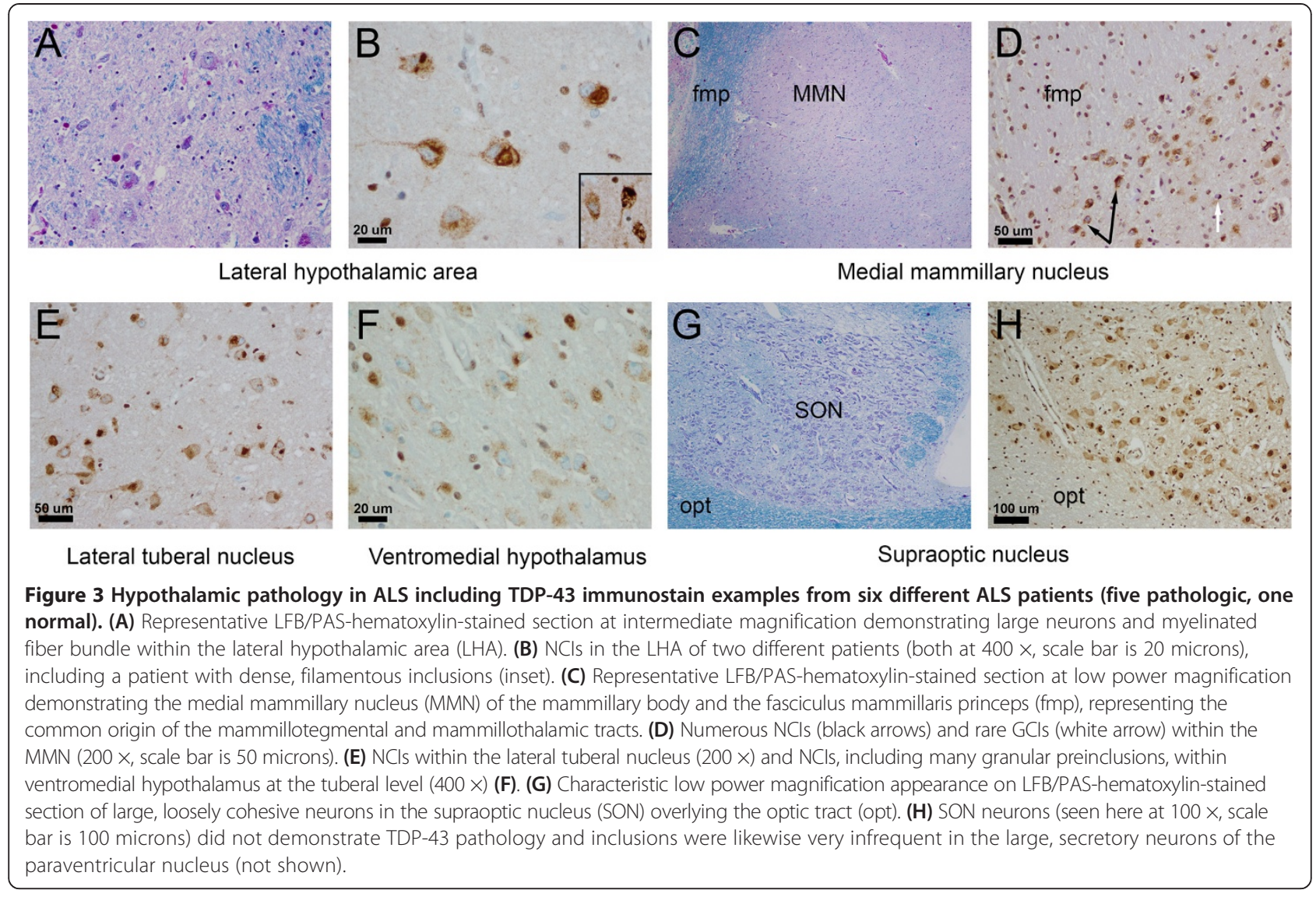

also did not significantly differ between patients with noncanonical (median of 3.0 years, interquartile range, 2.44.7) and canonical TDP-43 pathology (median of 3.1 years, interquartile range, 2.1-4.9) $(\mathrm{W}=109, \mathrm{p}=0.92)$. Patient age at death did not significantly differ between those with (mean $=64.1$ years, $\mathrm{sd}=11.33$ years) and without (mean $=62.0$ years, $\mathrm{sd}=7.96$ years) TDP-43 pathology in basal forebrain/hypothalamus ( $\mathrm{W}=95, \mathrm{p}=0.33)$.

\section{Association of basal forebrain and hypothalamic pathology with BMI}

BMI did not significantly differ between patients with (median BMI of $21.9 \mathrm{~kg} / \mathrm{m}^{2}$, interquartile range, 20.8 28.2) and without (median of $26.2 \mathrm{~kg} / \mathrm{m}^{2}$, interquartile range, 23.4-28.0) any basal forebrain and/or hypothalamic pathology $(\mathrm{W}=82, \mathrm{p}=0.57)$. BMI did significantly differ, however, between patients with (median of $20.9 \mathrm{~kg} / \mathrm{m}^{2}$, interquartile range, 20.7-21.3) and without (median of $27.1 \mathrm{~kg} / \mathrm{m}^{2}$, interquartile range, 24.1-28.2) pathology in the LHA specifically ( $\mathrm{W}=11, \mathrm{p}=0.023$ ) (see Figure 4). Both initial BMI (at a median interval of 8.9 months from symptom onset) and last recorded BMI (3.6 months from measurement until patient death, see Materials and methods for detail) were available in 25 patients. The percent differences between initial and last recorded BMI (positive percent difference values indicating loss of BMI points) were $6.0 \%, 8.3 \%$, and $26.6 \%$ for patients with no forebrain/hypothalamic TDP-43 pathology, any forebrain/ hypothalamic pathology, and LHA/TMN region TDP-43 pathology, respectively.

\section{Discussion}

Patients with ALS may experience a variety of extra-motor symptoms that have been referred to as "ALS-plus" syndromes, but that may simply represent a more complete spectrum of the disease [1]. These signs and symptoms include energy metabolism deficits [12], subclinical autonomic nervous system dysfunction [8], and frontal executive dysfunction manifesting as cognitive impairment in up to $51 \%$ of patients [5].

This study is the first to systematically investigate whether TDP-43 pathology in the basal forebrain and hypothalamus of ALS patients might contribute to these extra-motor symptoms, given the important roles of these regions in cognition, energy metabolism and thermoregulation, endocrine function, feeding behaviors, and autonomic responses [25,33,34]. TDP-43 pathology was identified in this region in one-third of patients and stereotypic involvement of certain structures was found (e.g., VS/Is, BNST, medium-sized neurons of SI, LHA/TMN, 


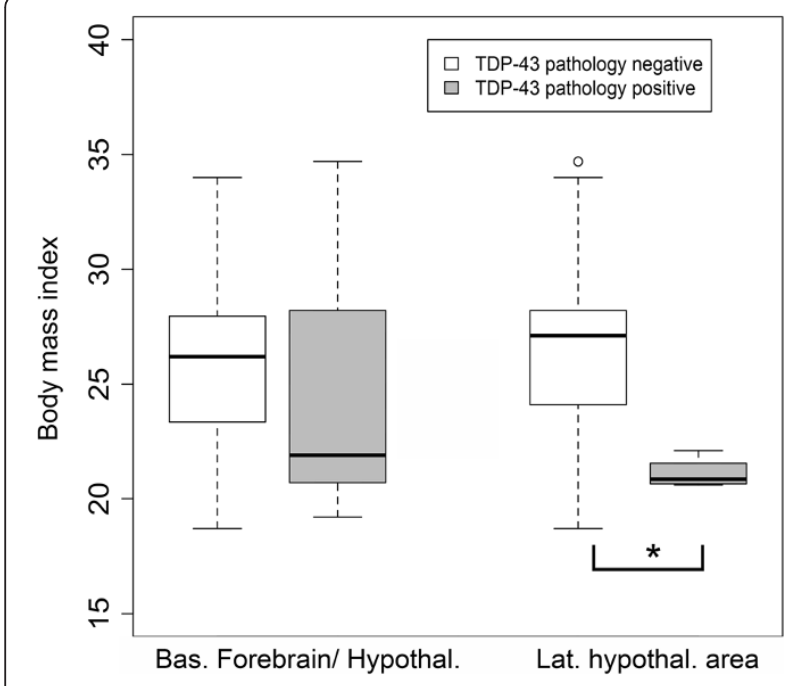

Figure 4 Boxplots of BMI relative to involvement of any region in the basal forebrain and/or hypothalamus (left) versus LHA (right) for patients with a BMI measure within $\leq 24$ months the time of death (median interval of $\mathbf{3 . 6}$ months). Plots represent BMl for basal forebrain/hypothalamus ( $n=29 ; 19$ patients without TDP-43 pathology and 10 with inclusion pathology) and for patients with the LHA sampled ( $n=25 ; 21$ patients without TDP-43 pathology and 4 with inclusion pathology). There was no significant difference in $\mathrm{BMI}$ for patients with and without TDP-43 pathology in any component of forebrain/hypothalamus (left). For the LHA, a significant difference in BMI was identified between patients with and without TDP-43 pathology $(p=0.02)$. Boxplot prepared in R (R Foundation for Statistical Computing, Vienna, Austria, 2014)

$\mathrm{MB} / \mathrm{LTN}$ ) with little or no involvement of other structures (VP, SON, PVN). Pathology in this region was seen only in the context of other non-canonical TDP-43, including within dorsal striatum, thalamus, non-frontal cortex, hippocampus (each of these regions involved in seven patients) and amygdala (in eight patients) (see Figure 5). No patients with isolated motor neuron and hypothalamic pathology were identified, and with a single exception, hypothalamic pathology appeared to follow basal forebrain pathology. As such, the pathology identified here is best placed in context of the progressive, widespread TDP43 proteinopathy recently characterized as stage III and stage IV for ALS TDP-43 pathology [35].

We also tested whether AD neuropathology [36] or aging [19] influenced the TDP-43 pathology identified. The lack of advanced AD neuropathology (see Table 1) and demonstration that age did not influence TDP-43 pathology in the cohort suggests this is not the case and that these findings are in indeed within the spectrum of ALS pathology.

\section{The location of forebrain and hypothalamic pathology in ALS}

The finding of basal forebrain and hypothalamic pathology in ALS patients is consistent with earlier observations. One recent study of pathologic staging in ALS identified the heaviest burden of subcortical TDP-43 pathology in the VS [35]. We similarly identified NCIs in VS, but also within the associated small granule cell islands adjacent to VS and within the area of the olfactory tubercle (Figure 2E, F). Though their role is poorly understood, these small neurons are thought to receive afferent input from serotonergic and catecholaminergic cells of the brainstem and may have projections to dendrites of adjacent striatal cells [37]. NCIs also were identified in the LSA (two patients) with both of these patients having filamentous or skein-like inclusions in magnocellular neurons of basal forebrain (nucleus of the diagonal band). This relationship is intriguing as a "hippocampal-septal area-diagonal band" circuit has been proposed as a structural subunit of basal forebrain organization [33] and raises the question of whether the pathology identified may reflect underlying structural and functional homologies between neuronal groups.

TDP-43 pathology was also identified in mediumsized, nondescript neurons of the SI that could not be attributed to VP, VS/Is, or magnocellular neuron groups (see Figure $2 \mathrm{H}$ ). The possibility that these cells represent extensions of amygdala into the basal forebrain [33] would be consistent with the frequent involvement of amygdala in ALS patients, as well as with frequent concurrent pathologic involvement of the BNST (also identified here), which itself is a portion of the "extended" amygdala [38]. TDP-43 pathology in amygdala and extended amygdala may in part underlie behavioral alterations in some ALS patients that are usually present in non-ALS patients with bilateral amygdala damage [39].

There are fewer examples of pathologic involvement of the hypothalamus in the ALS literature. Takahashi and colleagues reported a single case of ALS with cell loss and gliosis in cortical and subcortical neurons, including within the $\mathrm{MB}$ [40]. A recent study from Mayo Clinic Jacksonville identified TDP-43 NCIs in the hypothalamus of ALS and FTLD/ALS patients (more frequently in the latter group) [41]. We identified neuronal cytoplasmic TDP-43 inclusions in five patients, most frequently within neurons of the LHA (see Figure 3B), as well as within neurons of MB and adjacent tuberal nuclei. Neurons of hypothalamus, particularly the LHA, may receive both neural and neurohormonal input to regulate a number of functions including metabolic rate, feeding behavior, and autonomic function [34]. Further studies are also warranted to examine affected nuclei using both TDP-43 and proteins marking neuronal sub-populations (e.g., orexin/hypocretin, alpha-melanocyte stimulating hormone).

\section{Potential implications of forebrain and hypothalamic pathology in ALS}

The finding of significantly reduced BMI in patients with LHA pathology is intriguing (see Figures 3 and 4) and 


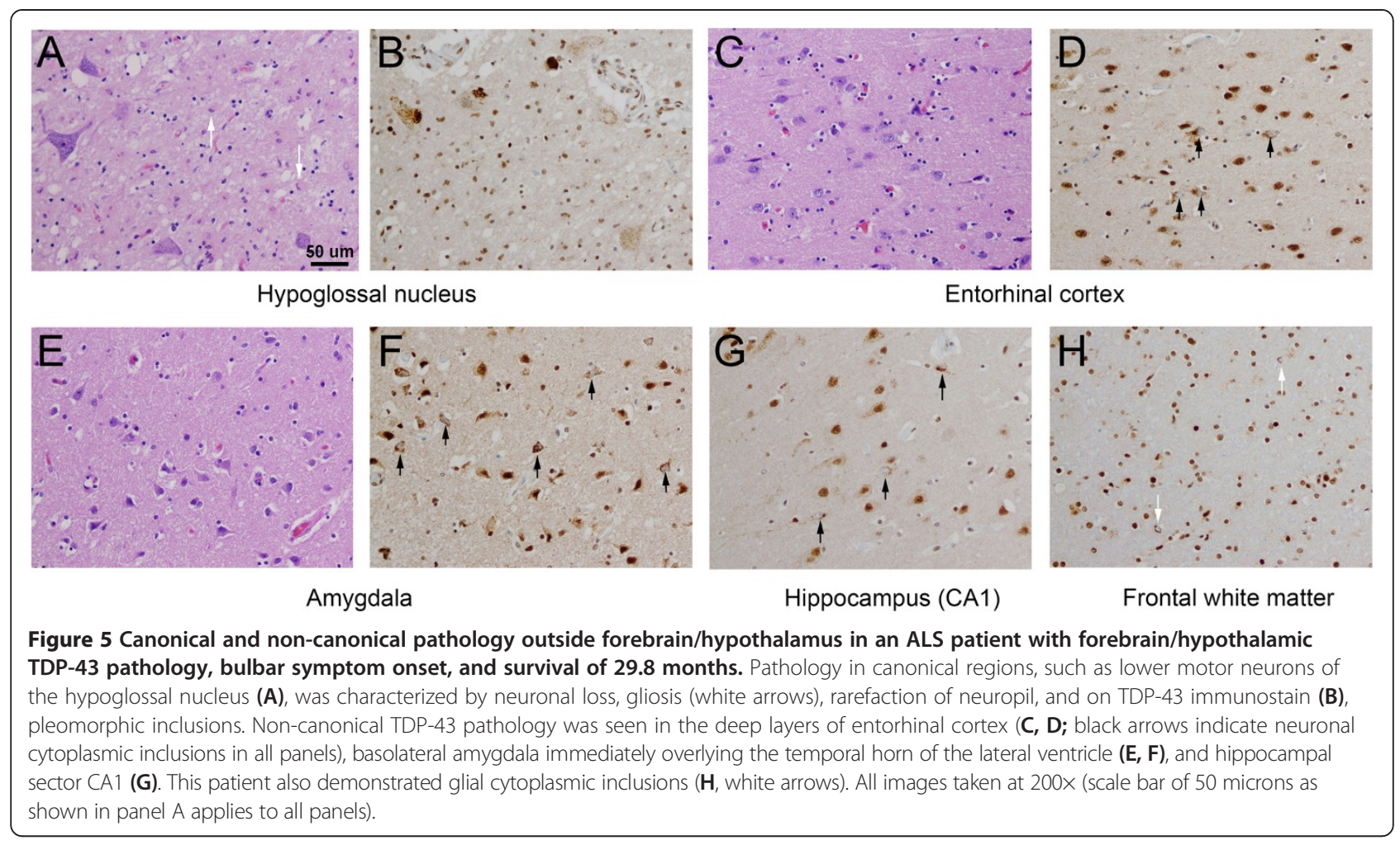

suggests the possibility that pathology in this region may contribute to metabolic disturbances and weight loss in ALS, in addition to increased muscle work, possible mitochondrial defects [12], and dysphagia/bulbar symptoms [42]. Patients with ALS have a paradoxical elevation in resting energy expenditure $(\sim 10 \%$ greater than matched controls), which is surprising given their loss of fat-free mass (itself an important determinant of basal metabolic rate) [43]. The pathophysiologic bases for this finding remain unexplained but may relate to nutritional status, which is prognostically important [13]. However, several caveats require mention. First, rare patients without forebrain and hypothalamic pathology had a relatively low last available BMI (see Figure 4), which indicates the mechanisms underlying metabolic alterations and BMI loss in ALS are multiple and not entirely understood. Second, a significant association of bulbar onset of symptoms and forebrain/hypothalamic pathology was identified. Even limb-onset patients often experience bulbar symptoms late in their disease course. Aggressive clinical interventions are performed in such patients to counteract the detrimental effects of dysphagia on BMI [42]; still, the exact contributions of pathologic and clinical factors on weight loss are difficult to estimate. An additional question is whether BMI changes in ALS patients are the result of extensive TDP-43 proteinopathy or the result of neuronal dysfunction in specific populations. We had identified forebrain and hypothalamic pathology only in patients with widespread TDP-43 inclusions (e.g., Figure 5). BMI did not significantly differ when any forebrain/hypothalamic pathology was considered (Figure 4, left box plots), which suggests the LHA region pathology may be important in this respect. However, this does not exclude the possibility that TDP-43 pathology in other brain regions influences metabolic rate and BMI in ALS.

There likely are several other clinical implications for patients with hypothalamic pathology in ALS since this structure subserves affective behaviors, autonomic function, sleep-wake cycles, thermoregulation, feeding and reproductive behaviors, water balance, and endocrine function [29-31]. Clinical studies of hypothalamic function in ALS are rare so the clinical correlates of the pathology are difficult to estimate. Nonetheless, reduced growth hormone has been reported in up to $52 \%$ of ALS patients [10]. Likewise, inappropriate secretion of antidiuretic hormone has also been documented [9], which raises the possibility of hypothalamic neuronal dysfunction (e.g., osmoreceptors). Significant pathology in the LHA is also likely to impair the ascending arousal system in ALS patients. LHA and forebrain nuclei receive afferents from monoamenergic neurons in the brainstem (e.g., locus ceruleus), among other sites, and have a profound influence on wakefulness via projections to cerebral cortex [44]. Ultimately, any anatomic focus of significant hypothalamic pathology is likely to have multiple effects 
given the integrative role of this structure [31]. This is reflected in widespread connections to hippocampus, corticomedial and basolateral amygdala, basal ganglia, and midbrain tegmentum and reticular formation [29,31,32], as well as thalamus, dorsal medulla, neurohypophysis, preganglionic neurons of spinal cord [29], and isocortex $[31,32,34]$. The implications of TDP-43 pathology in this region are further complicated by the fact that basal forebrain and hypothalamic neurons are in anatomic contiguity (e.g., septal area, diagnonal band, and rostral hypothalamus) [30], are interconnected (e.g., via medial forebrain bundle), and for hypothalamus, have internuclear short fiber connections [32].

\section{Extent of TDP-43 pathology and disease duration in ALS}

An important finding of this study is an asynchrony between disease extent and clinically determined disease duration. Similar results have been observed in other autopsy series [35] and in reports of patients with rapid disease progression yet widespread TDP-43 pathology [45]. These findings support a model of non-linear propagation of neuropathology in this disease that is not timedependent [46]. As such, pathology in ALS appears distinct from AD and Lewy body spectrum-diseases, in which extent and intensity of the tauopathy and alphasynucleinopathy, respectively, are presumed to be modified by disease duration with a spectrum of pre-clinical pathologic stages $[16,20]$.

Three patients in this study, for example, had unusually long survival ( $>6.5$ years), and their pathology was limited to canonical regions (spinal cord, brainstem, agranular frontal cortex). Conversely, three different patients with disease durations less than 2.5 years had extensive TDP-43 proteinopathy, including within the regions investigated in this study. The determinants of extent of TDP-43 pathology, of which disease duration does not appear to be one, therefore remain unclear. Age has been suggested as a negative prognostic factor in ALS, but our analysis shows this was not a determinant of the extent of TDP-43 pathology. Immune dysregulation may play a role as patients with "rapidly progressive" ALS have a reduction in regulatory $\mathrm{T}$-lymphocytes and reduction in transcription factors required for the function of this T-cell population (i.e., FoxP3) [47]. This suggests the intriguing possibility that short-lived patients with immune dysregulation may be among those with widespread TDP-43 deposition and rapid progression. Lastly, the significant association of basal forebrain and hypothalamic pathology with bulbar symptoms at onset identified here suggests the possibility that the site of disease initiation may play an important role in the propagation and final extent of disease. These and other factors that influence the kinetics and extent of TDP-43 spread are poorly understood and are deserving of further investigation.

\section{Study limitations}

There are limitations of the present study due to the retrospective design. First, the study was conducted with archived sections available or with supplemental sections that could be generated from stored tissues. In rare instances, involving older archived cases, one or more anatomic regions were not available in the original material and no additional tissue was available for sampling. Prospective sampling in future ALS cases will ensure more uniformity in the anatomic regions available for assessment. Based on our findings, two sections are likely to yield the most valuable pathologic data for future studies. The first, inclusive of basal forebrain, could be taken at level of the optic chiasm, anterior commissure, septal area, and BNST, and would demonstrate LSA, BNST, VS/Is, VP, and rostral hypothalamus (Figure 1A). The second, inclusive of tuberal and caudal hypothalamus, could be taken at the level of the tuber cinereum and fornix and would demonstrate LHA/TMN, portions of MB, LTN, periventricular nuclei of the tuberal region including $\mathrm{VMH}$, and the PHA (Figure 1B). Standardized sections across many non-ALS and ALS patients would also allow for more precise stereological analysis to determine the extent of neuronal loss, which is difficult to estimate by $\mathrm{H} \& \mathrm{E}$ and TDP-43 stains.

The ability to determine the clinical sequelae of the pathology described herein is also limited by the retrospective nature of the study. Future clinicopathologic studies of these clinical features with respect to the brain regions identified here will further clarify the importance of these pathologic findings in the extra-motor manifestations of ALS. This is particularly true with respect to measures of energy metabolism. The optimal clinical measures would include careful documentation of BMI at multiple time points [7] and measured resting energy expenditure [42] as calculated resting energy expenditure underestimates the hypermetabolism observed in ALS patients [43]. The pathologic findings documented herein provide a basis for the closer examination of these clinical measures with respect to TDP-43 pathology.

\section{Conclusions}

This study has demonstrated that a subset of patients with ALS have TDP-43 pathology within basal forebrain and hypothalamus, which may contribute to the extramotor symptoms of the disease, including alterations in energy metabolism, autonomic nervous system dysfunction, and frontal executive dysfunction. The pathologic involvement was fairly stereotypic, and in nearly all cases pathologic involvement of forebrain components (VS/Is, BNST, medium-sized neurons of SI) preceded hypothalamic involvement (LHA/TMN, MB/LTN). Basal forebrain and hypothalamic pathology was associated with the presence of other non-canonical TDP-43 inclusion 
pathology but was not associated with disease duration. Finally, the presence of forebrain/hypothalamic pathology was associated with bulbar onset of disease, which suggests the possibility of a unique and previously unrecognized clinico-pathological relationship in ALS.

\section{Ethical approval}

All procedures performed in studies involving human participants were in accordance with the ethical standards of the institutional and/or national research committee and with the 1964 Helsinki declaration and its later amendments or comparable ethical standards.

\section{Consent}

Written consent was obtained by the patients and/or their representatives to allow for their autopsy tissue to be used for research purposes. The study was also carried out with IRB approval from Houston Methodist Hospital.

\section{Competing interests}

The authors declare that they have no competing interests.

\section{Authors' contributions}

MDC and HT performed the main pathologic analyses described herein. SZP reviewed salient pathologic findings described here and performed additional pathologic analyses with MDC. HT, SZP, and AR (acknowledged) performed the primary autopsy examination in the patient's studied. MDC conceived of the study, performed the statistical analyses, and drafted the manuscript, figures, and tables. SHA provided clinical data as requested, as well as expert clinical input. PES provided expert clinical input to the project. All authors read, edited, and approved the final manuscript.

\section{Authors' information}

MDC is a second-year fellow in the Houston Methodist Hospital/M.D. Anderson Neuropathology Fellowship Program. HT is Medical Director of the Autopsy Service and Associate Medical Director of Neuropathology in the Department of Pathology and Genomic Medicine at Houston Methodist Hospital. SZP is Chief of Neuropathology in the Department of Pathology and Genomic Medicine at Houston Methodist Hospital where she also serves as co-director of the Neuropathology Fellowship Program. SHA is the Peggy and Gary Edwards Distinguished Endowed Chair in the Department of Neurology at Houston Methodist Hospital and the Director of the Houston Methodist Neurological Institute. PES is Professor in the Department of Neurology at the University of Texas Health Science Center at Houston and serves as Director in the Dementia Program at the Mischer Neuroscience Institute.

\section{Acknowledgements}

We are grateful for the excellent technical assistance of Ms. Candice Hamilton (Department of Pathology and Genomic Medicine, Section of Immunohistochemistry, Houston Methodist Hospital). We thank Andreana L. Rivera, MD (Department of Pathology and Genomic Medicine, Neuropathology section, Houston Methodist Hospital) who performed the primary autopsy examination in several patients studied here, as well as Ms. Karen Toennis RN, BSN (ALS Clinic Coordinator at Houston Methodist Hospital) who provided requested clinical information on select patients. This work was previously presented as an oral presentation at the $90^{\text {th }}$ annual meeting of the American Association of Neuropathologists (June 2014, Portland, OR).

\section{Author details}

'Department of Pathology and Genomic Medicine, Houston Methodist Hospital, 6565 Fannin Street, Suite M227, Houston, TX 77030, USA. ${ }^{2}$ Houston Methodist Neurological Institute, Houston Methodist Hospital, Houston, TX, USA. ${ }^{3}$ Department of Neurology, University of Texas Medical School at Houston, Houston, TX, USA. ${ }^{4}$ Mischer Neuroscience Institute, Houston, TX, USA. ${ }^{5}$ Department of Neurology, Houston Methodist Hospital, Houston, TX, USA.
Received: 26 November 2014 Accepted: 27 November 2014

Published online: 24 December 2014

\section{References}

1. Ravits J, Appel S, Baloh RH, Barohn R, Brooks BR, Elman L, Floeter MK, Henderson C, Lomen-Hoerth C, Macklis JD, McCluskey L, Mitsumoto H, Przedborski S, Rothstein J, Trojanowski JQ, van den Berg LH, Ringel S (2013) Deciphering amyotrophic lateral sclerosis: what phenotype, neuropathology and genetics are telling us about pathogenesis. Amyotroph Lateral Scler Frontotemporal Degener 14(Suppl 1):5-18, doi:10.3109/ 21678421.2013.778548

2. Al-Chalabi A, Jones A, Troakes C, King A, Al-Sarraj S, van den Berg LH (2012) The genetics and neuropathology of amyotrophic lateral sclerosis. Acta Neuropathol 124(3):339-352, doi:10.1007/s00401-012-1022-4

3. Geser F, Brandmeir NJ, Kwong LK, Martinez-Lage M, Elman L, McCluskey L, Xie SX, Lee VM, Trojanowski JQ (2008) Evidence of multisystem disorder in whole-brain map of pathological TDP-43 in amyotrophic lateral sclerosis. Arch Neurol 65(5):636-641, doi:10.1001/archneur.65.5.636

4. Zhang YJ, Xu YF, Cook C, Gendron TF, Roettges P, Link CD, Lin WL, Tong J Castanedes-Casey M, Ash P, Gass J, Rangachari V, Buratti E, Baralle F, Golde TE, Dickson DW, Petrucelli L (2009) Aberrant cleavage of TDP-43 enhances aggregation and cellular toxicity. Proc Natl Acad Sci U S A 106(18):76077612, doi:10.1073/pnas.0900688106

5. Ringholz GM, Appel SH, Bradshaw M, Cooke NA, Mosnik DM, Schulz PE (2005) Prevalence and patterns of cognitive impairment in sporadic ALS. Neurology 65(4):586-590, doi:10.1212/01.wnl.0000172911.39167.b6

6. Brettschneider J, Libon DJ, Toledo JB, Xie SX, McCluskey L, Elman L, Geser F, Lee VM, Grossman M, Trojanowski JQ (2012) Microglial activation and TDP-43 pathology correlate with executive dysfunction in amyotrophic lateral sclerosis. Acta Neuropathol 123(3):395-407, doi:10.1007/s00401-011-0932-x

7. Jawaid A, Murthy SB, Wilson AM, Qureshi SU, Amro MJ, Wheaton M, Simpson E, Harati Y, Strutt AM, York MK, Schulz PE (2010) A decrease in body mass index is associated with faster progression of motor symptoms and shorter survival in ALS. Amyotroph Lateral Scler 11(6):542-548, doi:10.3109/17482968.2010.482592

8. Baltadzhieva R, Gurevich T, Korczyn AD (2005) Autonomic impairment in amyotrophic lateral sclerosis. Curr Opin Neurol 18(5):487-493

9. Arakawa K, Nishio T, Sunohara N (1997) Two cases of MND/ALS developing the syndrome of inappropriate secretion of antidiuretic hormone. Acta Neurol Scand 96(4):262-265

10. Pellecchia MT, Pivonello R, Monsurro MR, Trojsi F, Longo K, Piccirillo G, Pivonello C, Rocco M, Di Somma C, Colao A, Tedeschi G, Barone P (2010) The GH-IGF system in amyotrophic lateral sclerosis: correlations between pituitary GH secretion capacity, insulin-like growth factors and clinical features. Eur J Neurol 17(5):666-671, doi:10.1111/j.1468-1331.2009.02896.x

11. Gonzalez de Aguilar JL, Gordon JW, Rene F, Lutz-Bucher B, Kienlen-Campard P, Loeffler JP (1999) A mouse model of familial amyotrophic lateral sclerosis expressing a mutant superoxide dismutase 1 shows evidence of disordered transport in the vasopressin hypothalamo-neurohypophysial axis. Eur J Neurosci 11(12):4179-4187

12. Dupuis L, Pradat PF, Ludolph AC, Loeffler JP (2011) Energy metabolism in amyotrophic lateral sclerosis. Lancet Neurol 10(1):75-82, doi:10.1016/S14744422(10)70224-6

13. Chio A, Logroscino G, Hardiman O, Swingler R, Mitchell D, Beghi E, Traynor BG (2009) Prognostic factors in ALS: A critical review. Amyotroph Lateral Scler 10(5-6):310-323, doi:10.3109/17482960802566824

14. Schultz C, Ghebremedhin E, Braak H, Braak E (1997) Neurofibrillary pathology in the human paraventricular and supraoptic nuclei. Acta Neuropathol 94(1):99-102

15. Braak H, Braak E (1989) Cortical and subcortical argyrophilic grains characterize a disease associated with adult onset dementia. Neuropathol Appl Neurobiol 15(1):13-26

16. Braak H, Del Tredici K, Rub U, de Vos RA, Jansen Steur EN, Braak E (2003) Staging of brain pathology related to sporadic Parkinson's disease. Neurobiol Aging 24(2):197-211

17. Benarroch EE, Schmeichel AM, Sandroni P, Low PA, Parisi JE (2007) Involvement of hypocretin neurons in multiple system atrophy. Acta Neuropathol 113(1):75-80, doi:10.1007/s00401-006-0150-0

18. Swaab DF, Hofman MA, Lucassen PJ, Purba JS, Raadsheer FC, Van de Nes JA (1993) Functional neuroanatomy and neuropathology of the human hypothalamus. Anat Embryol (Berl) 187(4):317-330 
19. Wilson RS, Yu L, Trojanowski JQ, Chen EY, Boyle PA, Bennett DA, Schneider JA (2013) TDP-43 pathology, cognitive decline, and dementia in old age. JAMA Neurol 70(11):1418-1424, doi:10.1001/jamaneurol.2013.3961

20. Braak H, Braak E (1991) Neuropathological stageing of Alzheimer-related changes. Acta Neuropathol 82(4):239-259

21. Montine TJ, Phelps CH, Beach TG, Bigio EH, Cairns NJ, Dickson DW, Duyckaerts C, Frosch MP, Masliah E, Mirra SS, Nelson PT, Schneider JA, Thal DR, Trojanowski JQ, Vinters HV, Hyman BT (2012) National Institute on Aging-Alzheimer's Association guidelines for the neuropathologic assessment of Alzheimer's disease: a practical approach. Acta Neuropathol 123(1):1-11, doi:10.1007/s00401-011-0910-3

22. Mirra SS, Heyman A, McKeel D, Sumi SM, Crain BJ, Brownlee LM, Vogel FS, Hughes JP, van Belle G, Berg L (1991) The Consortium to Establish a Registry for Alzheimer's Disease (CERAD). Part II. Standardization of the neuropathologic assessment of Alzheimer's disease. Neurology 41(4):479-486

23. Thal DR, Rub U, Orantes M, Braak H (2002) Phases of A beta-deposition in the human brain and its relevance for the development of AD. Neurology 58(12):1791-1800

24. Dickson D, Weller RO (2011) Neurodegeneration: the molecular pathology of dementia and movement disorders, 2nd edn. International Society of Neuropathology, Chichester, West Sussex, Wiley-Blackwell

25. Haber S (1987) Anatomical relationship between the basal ganglia and the basal nucleus of Meynert in human and monkey forebrain. Proc Natl Acad Sci U S A 84(5):1408-1412

26. Ulfig N, Braak E, Ohm TG, Pool CW (1990) Vasopressinergic neurons in the magnocellular nuclei of the human basal forebrain. Brain Res 530(1):176-180

27. Crosby EC, Humphrey T (1941) Studies of the vertebrate telencephalon. II. The nuclear pattern of the anterior olfactory nucleus, tuberculum olfactorium and the amygdaloid complex in adult man. J Comp Neurol 74(2):309-352

28. Prensa L, Richard S, Parent A (2003) Chemical anatomy of the human ventral striatum and adjacent basal forebrain structures. J Comp Neurol 460(3):345-367, doi:10.1002/cne.10627

29. Carpenter MB (1985) Core text of neuroanatomy, 3rd edn. Williams \& Wilkins, Baltimore

30. Braak H, Braak E (1987) The hypothalamus of the human adult: chiasmatic region. Anat Embryol (Berl) 175(3):315-330

31. Saper CB (2012) Hypothalamus. In: Mai JK, Paxinos G (eds) The human nervous system, 3rd edn. Elsevier Academic Press, Amsterdam; Boston, pp $548-583$

32. Crosby EC, National Institute on Drug Abuse, Addiction Research Center (U.S.) (1962) Correlative anatomy of the nervous system. MacMillan, New York

33. Alheid GF (2003) Extended amygdala and basal forebrain. Ann N Y Acad Sci 985:185-205

34. Berthoud HR, Munzberg H (2011) The lateral hypothalamus as integrator of metabolic and environmental needs: from electrical self-stimulation to opto-genetics. Physiol Behav 104(1):29-39, doi:10.1016/j.physbeh.2011.04.051

35. Brettschneider J, Del Tredici K, Toledo JB, Robinson JL, Irwin DJ, Grossman M, Suh E, Van Deerlin VM, Wood EM, Baek Y, Kwong L, Lee EB, Elman L, McCluskey L, Fang L, Feldengut S, Ludolph AC, Lee VM, Braak H, Trojanowski JQ (2013) Stages of pTDP-43 pathology in amyotrophic lateral sclerosis. Ann Neurol 74(1):20-38, doi:10.1002/ana.23937

36. Josephs KA, Murray ME, Whitwell JL, Parisi JE, Petrucelli L, Jack CR, Petersen RC, Dickson DW (2014) Staging TDP-43 pathology in Alzheimer's disease. Acta Neuropathol 127(3):441-450, doi:10.1007/s00401-013-1211-9

37. Haber SN, Adler A, Bergman H (2012) The Basal Ganglia. In: Mai JK, Paxinos $\mathrm{G}$ (eds) The human nervous system, 3rd edn. Elsevier Academic Press, Amsterdam; Boston, pp 678-738

38. Yilmazer-Hanke DM (2012) Amygdala. In: Mai JK, Paxinos G (eds) The human nervous system, 3rd edn. Elsevier Academic Press, Amsterdam; Boston, pp 759-835

39. Schmolck H, Mosnik D, Schulz P (2007) Rating the approachability of faces in ALS. Neurology 69(24):2232-2235, doi:10.1212/01.wnl.0000296001.16603.b3

40. Takahashi T, Yagishita S, Amano N, Yamaoka K, Kamei T (1997) Amyotrophic lateral sclerosis with numerous axonal spheroids in the corticospinal tract and massive degeneration of the cortex. Acta Neuropathol 94(3):294-299

41. Murray ME, DeJesus-Hernandez M, Rutherford NJ, Baker M, Duara R, Graff-Radford NR, Wszolek ZK, Ferman TJ, Josephs KA, Boylan KB, Rademakers R, Dickson DW (2011) Clinical and neuropathologic heterogeneity of c9FTD/ALS associated with hexanucleotide repeat expansion in C9ORF72. Acta Neuropathol 122(6):673-690, doi:10.1007/s00401-011-0907-y
42. Desport JC, Preux PM, Truong CT, Courat L, Vallat JM, Couratier P (2000) Nutritional assessment and survival in ALS patients. Amyotroph Lateral Scler Other Motor Neuron Disord 1(2):91-96

43. Desport JC, Preux PM, Magy L, Boirie Y, Vallat JM, Beaufrere B, Couratier P (2001) Factors correlated with hypermetabolism in patients with amyotrophic lateral sclerosis. Am J Clin Nutr 74(3):328-334

44. Saper CB, Scammell TE, Lu J (2005) Hypothalamic regulation of sleep and circadian rhythms. Nature 437(7063):1257-1263, doi:10.1038/nature04284

45. McCluskey LF, Elman LB, Martinez-Lage M, Van Deerlin V, Yuan W, Clay D, Siderowf A, Trojanowski JQ (2009) Amyotrophic lateral sclerosis-plus syndrome with TAR DNA-binding protein-43 pathology. Arch Neurol 66(1):121-124, doi:10.1001/archneur.66.1.121

46. Ravits J (2014) Focality, stochasticity and neuroanatomic propagation in ALS pathogenesis. Exp Neurol doi:10.1016/j.expneurol.2014.07.021

47. Henkel JS, Beers DR, Wen S, Rivera AL, Toennis KM, Appel JE, Zhao W, Moore DH, Powell SZ, Appel SH (2013) Regulatory T-lymphocytes mediate amyotrophic lateral sclerosis progression and survival. EMBO Mol Med 5 (1):64-79, doi:10.1002/emmm.201201544

\section{Submit your next manuscript to BioMed Central and take full advantage of:}

- Convenient online submission

- Thorough peer review

- No space constraints or color figure charges

- Immediate publication on acceptance

- Inclusion in PubMed, CAS, Scopus and Google Scholar

- Research which is freely available for redistribution 